\title{
Spectral properties of quotients of Beurling-type submodules of the Hardy module over the unit ball
}

\author{
by \\ Kunyu Guo and YongJiang Duan (Shanghai)
}

\begin{abstract}
Let $M$ be a Beurling-type submodule of $H^{2}\left(\mathbb{B}_{d}\right)$, the Hardy space over the unit ball $\mathbb{B}_{d}$ of $\mathbb{C}^{d}$, and let $N=H^{2}\left(\mathbb{B}_{d}\right) / M$ be the associated quotient module. We completely describe the spectrum and essential spectrum of $N$, and related index theory.
\end{abstract}

1. Introduction. Let $\mathbb{B}_{d}$ be the unit ball of $\mathbb{C}^{d}$, and let $H^{2}\left(\mathbb{B}_{d}\right)$ be the Hardy space over $\mathbb{B}_{d}$, which consists of the analytic functions in $\mathbb{B}_{d}$ satisfying

$$
\sup _{0<r<1} \int_{\partial \mathbb{B}_{d}}|f(r \xi)|^{2} d \sigma(\xi)<\infty
$$

where $d \sigma$ is the normalized Lebesgue measure on the unit sphere $\partial \mathbb{B}_{d}$. Let $\eta$ be an inner function on the unit ball, that is, $\eta$ is a nonconstant function in $H^{\infty}\left(\mathbb{B}_{d}\right)$ satisfying $\left|f^{*}(\zeta)\right|=1$ a.e. $[\sigma]$ on $\partial \mathbb{B}_{d}$, where $f^{*}(\zeta)=\lim _{r \rightarrow 1} f(r \zeta)$. In the sixties, Rudin posed the existence problem: Do there exist inner functions in $H^{\infty}\left(\mathbb{B}_{d}\right)$ [Rud1]? This problem was affirmatively solved in 1982 by B. Aleksandrov [Rud2].

When $d=1, \mathbb{B}_{1}=U$ is the open unit disk, and $H^{2}(U)$ is the classical Hardy space over $U$. Let $N=H^{2}(U) \ominus \eta H^{2}(U)$ for a one-variable inner function $\eta$, and let $N_{z}=P_{N} M_{z} \mid N$ be the compression of the coordinate multiplication operator $M_{z}$ to $N$; here $P_{N}$ is the projection onto $N$. Then the Livšic-Moeller theorem [Nil, p. 62] states that the spectrum of $N_{z}$ equals the zero set of $\eta$ in the open unit disk, together with the points on the unit circle $\mathbb{T}$ to which $\eta$ cannot be analytically continued from $U$, that is,

$$
\sigma\left(N_{z}\right)=Z(\eta) \cup E
$$

where $Z(\eta)$ is the zero set of $\eta$ in the unit disk and

$E=\left\{\lambda \in \mathbb{T} \mid\right.$ there is a sequence $\left\{\xi_{i}\right\} \subset U$ such that

$$
\left.\lim _{i \rightarrow \infty} \xi_{i}=\lambda \text { and } \lim _{i \rightarrow \infty} \eta\left(\xi_{i}\right)=0\right\} .
$$

2000 Mathematics Subject Classification: 47A13, 47A20, 46H25, 46C99.

Key words and phrases: Beurling-type submodule, spectrum, essential spectrum.

This work is partially supported by NSF (10525106) and Young Teachers Fund. 
Moreover, if $\eta$ is not a finite Blaschke product, then a result of Arveson [Arv1, Theorem 3.4.3] shows that the essential spectrum of $N_{z}$ is exactly $E$. The proofs of these results rely heavily on the fact that each inner function $\eta$ $(d=1)$ has the factorization $\eta=B S$, where $B$ is a Blaschke product and $S$ is a singular inner function. When $d>1$, inner functions have no such factorization. In fact, the structure of inner functions in several variables is far from clear. In this paper, we will deal with the case $d>1$.

Given a Hilbert space $H$, let $\left(T_{1}, \ldots, T_{d}\right)$ be a tuple of commuting bounded operators acting on $H$. Then one naturally makes $H$ into a Hilbert module over the polynomial ring $\mathbb{C}\left[z_{1}, \ldots, z_{d}\right]$ (cf. $[\mathrm{DP}]$ ) by setting

$$
p \cdot \xi=p\left(T_{1}, \ldots, T_{d}\right) \xi, \quad p \in \mathbb{C}\left[z_{1}, \ldots, z_{d}\right], \xi \in H .
$$

Now let $\left(M_{z_{1}}, \ldots, M_{z_{d}}\right)$ be the coordinate multiplication operators acting on $H^{2}\left(\mathbb{B}_{d}\right)$. Then $H^{2}\left(\mathbb{B}_{d}\right)$ naturally becomes a Hilbert module over the polynomial ring $\mathbb{C}\left[z_{1}, \ldots, z_{d}\right]$, called the Hardy module on the unit ball $\mathbb{B}_{d}$. A closed subspace $M$ of $H^{2}\left(\mathbb{B}_{d}\right)$ is called a submodule if $p M \subset M$ for any polynomial $p$. In the case of one variable, Beurling's theorem shows that each submodule has the form $M=\eta H^{2}(U)$ for some inner function $\eta$. However, in the case of several variables, there exist a lot of submodules that do not have the above form, for example, submodules of finite codimension $[\mathrm{CG}, \mathrm{DP}]$. Given an inner function $\eta$ on $\mathbb{B}_{d}$, the submodule $M=\eta H^{2}\left(\mathbb{B}_{d}\right)$ is said to be a Beurling-type submodule. Given a submodule $M$ of $H^{2}\left(\mathbb{B}_{d}\right)$, the quotient module $H^{2}\left(\mathbb{B}_{d}\right) / M$ is naturally identified with $M^{\perp}$, where the module action on $M^{\perp}$ is $p \cdot \xi=P_{M^{\perp}} p \xi$ for any polynomial $p$ and $\xi \in M^{\perp}$.

This paper mainly considers spectral properties of quotients of Beurling-type submodules on the unit ball. Given an inner function $\eta$, let $N=H^{2}\left(\mathbb{B}_{d}\right) \ominus \eta H^{2}\left(\mathbb{B}_{d}\right)$ be the associated quotient module, and $N_{z}=$ $\left(N_{z_{1}}, \ldots, N_{z_{d}}\right)$ be the tuple of compression operators acting on $N$, where $N_{z_{i}}=P_{N} M_{z_{i}} \mid N$, and $P_{N}$ is the projection onto $N$. We will determine the spectrum and essential spectrum of the quotient module, that is, the Taylor spectrum and essential spectrum of the tuple $N_{z}=\left(N_{z_{1}}, \ldots, N_{z_{d}}\right)$. Determining the spectrum and essential spectrum of a general quotient module is very difficult since the problem is related to essential normality of the quotient module [Arv5, Arv6, Dou, GW]. But even for submodules generated by homogeneous polynomials, essential normality of submodules remains unknown [Arv5, Arv6, Dou]. The paper also concerns $K$-homology defined by quotient modules.

2. The Taylor spectrum of $N_{z}$. In this section, we will determine the spectrum of the quotient module $N$, i.e. the Taylor spectrum of $N_{z}=$ $\left(N_{z_{1}}, \ldots, N_{z_{d}}\right)$. First, let us recall the concept of the Taylor spectrum [Tay]. 
For $1 \leq k \leq d$, let

$$
I_{k}=\left\{\left(i_{1}, \ldots, i_{k}\right) \in \mathbb{N} \mid 1 \leq i_{1}<\cdots<i_{k} \leq d\right\} .
$$

Let $\left\{e_{i} \mid 1 \leq i \leq d\right\}$ be an orthonormal basis for $\mathbb{C}^{d}$, and $\bigwedge^{k} \mathbb{C}^{d}$ be the $k$ th exterior power of $\mathbb{C}^{d}$ with orthonormal basis $\left\{e_{i_{1}} \wedge \cdots \wedge e_{i_{k}} \mid\left(i_{1}, \ldots, i_{k}\right) \in I_{k}\right\}$. Let $\bigwedge \mathbb{C}^{d}=\bigoplus_{k=0}^{d} \bigwedge^{k} \mathbb{C}^{d}$, where $\bigwedge^{0} \mathbb{C}^{d}=\mathbb{C}$. Then $\bigwedge \mathbb{C}^{d}$ is a Hilbert space with orthonormal basis $\{1\} \cup\left\{e_{i_{1}} \wedge \cdots \wedge e_{i_{k}} \mid\left(i_{1}, \ldots, i_{k}\right) \in I_{k}, 1 \leq k \leq d\right\}$. Define the canonical creation operators $C_{1}, \ldots, C_{d}$ on $\wedge \mathbb{C}^{d}$ as follows:

$$
C_{i}: \xi \mapsto e_{i} \wedge \xi, \quad \xi \in \wedge \mathbb{C}^{d}
$$

Let $T_{1}, \ldots, T_{d}$ be a commuting $d$-tuple of operators on a Hilbert space $H$. Then the Koszul complex for the $d$-tuple $\left(T_{1}, \ldots, T_{d}\right)$ is

$$
0 \rightarrow \Omega^{0} \rightarrow \Omega^{1} \rightarrow \cdots \rightarrow \Omega^{d} \rightarrow 0, \quad \text { where } \quad \Omega^{k}=H \otimes \bigwedge^{k} \mathbb{C}^{d}
$$

with cohomological boundary operator

$$
B=T_{1} \otimes C_{1}+\cdots+T_{d} \otimes C_{d} .
$$

It is easy to see $B^{2}=0$. We denote the restriction of $B$ to $\Omega^{k}$ by $B_{k}$, and hence ran $B_{k-1} \subseteq$ ker $B_{k}$. Say that the tuple $\left(T_{1}, \ldots, T_{d}\right)$ is invertible if $\operatorname{ran} B_{k-1}=\operatorname{ker} B_{k}$, and $\left(T_{1}, \ldots, T_{d}\right)$ is Fredholm if

$$
\operatorname{dim}\left(\operatorname{ker} B_{k} / \operatorname{ran} B_{k-1}\right)<\infty \quad \text { for } 1 \leq k \leq d .
$$

When $T=\left(T_{1}, \ldots, T_{d}\right)$ is Fredholm, define its Fredholm index as

$$
\operatorname{ind}(T)=\sum_{k=1}^{d}(-1)^{k+1} \operatorname{dim}\left(\operatorname{ker} B_{k} / \operatorname{ran} B_{k-1}\right) .
$$

The Taylor spectrum and essential spectrum of $T=\left(T_{1}, \ldots, T_{d}\right)$ are defined as

$$
\begin{aligned}
\sigma(T) & =\left\{\left(\lambda_{1}, \ldots, \lambda_{d}\right) \in \mathbb{C}^{d} \mid\left(T_{1}-\lambda_{1}, \ldots, T_{d}-\lambda_{d}\right) \text { is not invertible }\right\}, \\
\sigma_{\mathrm{e}}(T) & =\left\{\left(\lambda_{1}, \ldots, \lambda_{d}\right) \in \mathbb{C}^{d} \mid\left(T_{1}-\lambda_{1}, \ldots, T_{d}-\lambda_{d}\right) \text { is not Fredholm }\right\} .
\end{aligned}
$$

We begin with a lemma which may be viewed as the $H^{\infty}$ functional calculus of $N_{z}$. It is a generalization of the one-variable version in [Nil], and the proof is omitted.

Lemma 2.1. Let

$$
\varphi\left(N_{z_{1}}, \ldots, N_{z_{d}}\right)=P_{N} \varphi\left|N, \quad \varphi\left(N_{z}^{*}\right)=P_{+} \bar{\varphi}^{t}\right| N,
$$

where $\varphi \in H^{\infty}, \varphi^{t}=\overline{\varphi\left(\bar{z}_{1}, \ldots, \bar{z}_{d}\right)}, N_{z}^{*}=\left(N_{z_{1}}^{*}, \ldots, N_{z_{d}}^{*}\right)$, and $P_{+}$is the projection from $L^{2}\left(\partial \mathbb{B}_{d}\right)$ onto $H^{2}\left(\mathbb{B}_{d}\right)$. Then

(1) $\left\|\varphi\left(N_{z}\right)\right\| \leq\|\varphi\|_{\infty}$; 
(2) the map $\varphi \mapsto \varphi\left(N_{z}\right)$ is linear, multiplicative, and

$$
P\left(N_{z}\right)=\sum_{\alpha} a_{\alpha} N_{z_{1}}^{\alpha_{1}} \cdots N_{z_{d}}^{\alpha_{d}}
$$

for each $P=\sum_{\alpha} a_{\alpha} z^{\alpha} \in \mathbb{C}\left[z_{1}, \ldots, z_{d}\right]$; moreover, $\varphi\left(N_{z}^{*}\right)=\varphi^{t}\left(N_{z}\right)^{*}$.

Proposition 2.2. $\sigma\left(N_{z}\right) \cap \mathbb{B}_{d}=Z(\eta)$, where $Z(\eta)=\left\{\lambda \in \mathbb{B}_{d} \mid \eta(\lambda)=0\right\}$ is the zero set of $\eta$.

Proof. From [Rud1, p. 116], one can solve Gleason's problem in $H^{\infty}\left(\mathbb{B}_{d}\right)$, that is, given $f \in H^{\infty}\left(\mathbb{B}_{d}\right)$ and $\lambda \in \mathbb{B}_{d}$, there exist $g_{1}, \ldots, g_{d} \in H^{\infty}\left(\mathbb{B}_{d}\right)$ such that

$$
f(z)-f(\lambda)=\sum_{i=1}^{d}\left(z_{i}-\lambda_{i}\right) g_{i}
$$

Replacing $f$ by $\eta$ and using the $H^{\infty}$ functional calculus for $N_{z}$, we have

$$
\eta\left(N_{z}\right)-\eta(\lambda)=\sum_{i=1}^{d}\left(N_{z_{i}}-\lambda_{i}\right) g_{i}\left(N_{z}\right) .
$$

Lemma 2.1 implies $\eta\left(N_{z}\right)=0$. Hence, if $\eta(\lambda) \neq 0$, then the tuple $N_{z}$ is invertible relative to $\left(N_{z}\right)^{\prime}$, the commutant algebra of the norm closed algebra generated by $N_{z_{1}}, \ldots, N_{z_{d}}$. This means $\sigma^{\prime}\left(N_{z}\right) \cap \mathbb{B}_{d} \subseteq Z(\eta)$, where $\sigma^{\prime}\left(N_{z}\right)$ denotes the algebra spectrum relative to the commutant algebra $\left(N_{z}\right)^{\prime}$ [Cur2]. By [Cur2, Lemma 4.5], $\sigma\left(N_{z}\right) \subseteq \sigma^{\prime}\left(N_{z}\right)$. This gives

$$
\sigma\left(N_{z}\right) \cap \mathbb{B}_{d} \subseteq Z(\eta) .
$$

To prove the reverse inclusion, let $\lambda \in Z(\eta)$. It suffices to show that

$$
N \neq\left(N_{z_{1}}-\lambda_{1}\right) N+\cdots+\left(N_{z_{d}}-\lambda_{d}\right) N
$$

since this implies that $\lambda \in \sigma\left(N_{z}\right)$. Indeed, assume that

$$
N=\left(N_{z_{1}}-\lambda_{1}\right) N+\cdots+\left(N_{z_{d}}-\lambda_{d}\right) N
$$

that is,

$$
H^{2}\left(\mathbb{B}_{d}\right) \ominus \eta H^{2}\left(\mathbb{B}_{d}\right)=P_{N}\left(\sum_{i=1}^{d}\left(M_{z_{i}}-\lambda_{i}\right)\left(H^{2}\left(\mathbb{B}_{d}\right) \ominus \eta H^{2}\left(\mathbb{B}_{d}\right)\right)\right) .
$$

Then, for each $g \in N=H^{2}\left(\mathbb{B}_{d}\right) \ominus \eta H^{2}\left(\mathbb{B}_{d}\right)$, there exist $f_{1}, \ldots, f_{d} \in$ $H^{2}\left(\mathbb{B}_{d}\right) \ominus \eta H^{2}\left(\mathbb{B}_{d}\right)$ and $h \in H^{2}\left(\mathbb{B}_{d}\right)$ such that

$$
h=\left(z_{1}-\lambda_{1}\right) f_{1}+\cdots+\left(z_{d}-\lambda_{d}\right) f_{d}, \quad g=P_{N} h .
$$

Hence, there exists $g_{1} \in H^{2}\left(\mathbb{B}_{d}\right)$ such that $h=\eta g_{1}+g$. Thus, $g=h-\eta g_{1}$, and so

$$
g(\lambda)=h(\lambda)-\eta(\lambda) g_{1}(\lambda)=0
$$


for each $g \in N=H^{2}\left(\mathbb{B}_{d}\right) \ominus \eta H^{2}\left(\mathbb{B}_{d}\right)$, which is obviously not true: if we decompose 1 as

$$
1=\eta f_{1}+f_{2},
$$

where $f_{1} \in H^{2}\left(\mathbb{B}_{d}\right)$ and $f_{2} \in N$, then obviously $f_{2}(\lambda) \neq 0$. This contradiction completes the proof of Proposition 2.2.

In the next section, we will prove that $\sigma_{\mathrm{e}}\left(N_{z}\right)=\partial \mathbb{B}_{d}$. Since $\sigma_{\mathrm{e}}\left(N_{z}\right)$ $\subseteq \sigma\left(N_{z}\right)$, it follows that $\sigma\left(N_{z}\right) \cap \partial \mathbb{B}_{d}=\partial \mathbb{B}_{d}$. Here, we want to give an elementary proof of this result.

Proposition 2.3. $\sigma\left(N_{z}\right) \cap \partial \mathbb{B}_{d}=\partial \mathbb{B}_{d}$.

We need the following lemma which comes from [Rud2, Theorem 1.2].

\section{Lemma 2.4. Suppose that:}

- $\Gamma$ is a nonempty open set in $\partial \mathbb{B}_{d}$;

- $\left\{r_{j}\right\}$ is a sequence satisfying $0 \leq r_{j}<1, r_{j} \nearrow 1$ as $j \rightarrow \infty$;

- $f \in H^{\infty}\left(\mathbb{B}_{d}\right), f$ is nonconstant, $\left|f^{*}(\zeta)\right|=1$ a.e. on $\Gamma$.

Then $\Gamma$ has a dense subset $H$ such that the set

$$
\left\{f\left(r_{j} \zeta\right) \mid j=1,2,3, \ldots\right\}
$$

is dense in the unit disk $U$ for every $\zeta \in H$.

For $f \in L^{\infty}\left(\partial \mathbb{B}_{d}\right)$, define the Toeplitz operator $T_{f}$ with symbol $f$ as

$$
T_{f}: H^{2}\left(\mathbb{B}_{d}\right) \rightarrow H^{2}\left(\mathbb{B}_{d}\right), \quad T_{f} h=P_{+} f h, \quad h \in H^{2}\left(\mathbb{B}_{d}\right),
$$

where $P_{+}$is the projection from $L^{2}\left(\partial \mathbb{B}_{d}\right)$ onto $H^{2}\left(\mathbb{B}_{d}\right)$.

Proof of Proposition 2.3. Applying Lemma 2.4 to $\partial \mathbb{B}_{d}$, with $f$ replaced by the inner function $\eta$, we get a dense subset $H$ of $\partial \mathbb{B}_{d}$ such that for each $\lambda \in H$, there is a sequence $\left\{\lambda_{j}\right\} \subseteq \mathbb{B}_{d}$ with $\lambda_{j} \rightarrow \lambda$ and $\eta\left(\lambda_{j}\right) \rightarrow 0$ as $j \rightarrow \infty$. Since $\sigma\left(N_{z}\right)$ is a compact set, it follows that $\sigma\left(N_{z}\right) \cap \partial \mathbb{B}_{d}$ is a closed subset of $\partial \mathbb{B}_{d}$. It is enough to prove that

$$
H \subseteq \sigma\left(N_{z}\right) \cap \partial \mathbb{B}_{d} .
$$

Indeed, assume there exists $\lambda_{0}=\left(\lambda_{01}, \ldots, \lambda_{0 d}\right) \in H$, but $\lambda_{0} \notin \sigma\left(N_{z}\right)$. Set $N_{z}^{*}=\left(N_{z_{1}}^{*}, \ldots, N_{z_{d}}^{*}\right)$. It follows from [Cur1, Corollary 3.14] that

$$
\sigma\left(N_{z}^{*}\right)=\left\{\bar{\lambda} \mid \lambda \in \sigma\left(N_{z}\right)\right\}
$$

Thus $\bar{\lambda}_{0} \notin \sigma\left(N_{z}^{*}\right)$. Recall

$$
\sigma_{\pi}\left(N_{z}^{*}\right)=\left\{\lambda \in \mathbb{C}^{d} \mid N_{z}^{*}-\lambda \text { is not jointly bounded below }\right\}
$$

(a tuple $\left(T_{1}, \ldots, T_{d}\right)$ on a Hilbert space $X$ is said to be jointly bounded below if there exists $\varepsilon>0$ such that $\sum_{i=1}^{d}\left\|T_{i} x\right\| \geq \varepsilon\|x\|$ for all $x \in X$.) Since $\sigma_{\pi}\left(N_{z}^{*}\right) \subseteq \sigma\left(N_{z}^{*}\right)$ (cf. [Cur2, p. 37]), this implies $\bar{\lambda}_{0} \notin \sigma_{\pi}\left(N_{z}^{*}\right)$, that is, 
there exists $\varepsilon_{0}>0$ such that for all $f \in N$,

$$
\sum_{i=1}^{d}\left\|\left(N_{z_{i}}^{*}-\bar{\lambda}_{0 i}\right) f\right\|=\sum_{i=1}^{d}\left\|\left(M_{z_{i}}^{*}-\bar{\lambda}_{0 i}\right) f\right\| \geq \varepsilon_{0}\|f\| .
$$

Now, we use (2.1) to prove that there exists $\delta>0$ such that

$$
\sum_{i=1}^{d}\left\|\left(M_{z_{i}}^{*}-\bar{\lambda}_{0 i}\right) g\right\|+\left\|T_{\bar{\eta}} g\right\| \geq \delta\|g\|, \quad \forall g \in H^{2}\left(\mathbb{B}_{d}\right) .
$$

For $g \in H^{2}\left(\mathbb{B}_{d}\right)$, write $g=h_{1}+\eta h_{2}$, where $h_{1} \in N$ and $h_{2} \in H^{2}\left(\mathbb{B}_{d}\right)$. It is easy to see that $T_{\bar{\eta}} h_{1}=0$, and thus

$$
T_{\bar{\eta}} g=T_{\bar{\eta}} h_{1}+T_{\bar{\eta}} \eta h_{2}=h_{2} \text {. }
$$

We have

$$
\begin{aligned}
& \sum_{i=1}^{d}\left\|\left(M_{z_{i}}^{*}-\bar{\lambda}_{0 i}\right) g\right\|+\left\|T_{\bar{\eta}} g\right\| \\
&=\sum_{i=1}^{d}\left\|\left(M_{z_{i}}^{*}-\bar{\lambda}_{0 i}\right) h_{1}+\left(M_{z_{i}}^{*}-\bar{\lambda}_{0 i}\right) \eta h_{2}\right\|+\left\|h_{2}\right\| .
\end{aligned}
$$

Let $A=\max _{1 \leq i \leq d}\left\|M_{z_{i}}^{*}-\bar{\lambda}_{0 i}\right\|>0$, and consider the following two cases:

CASE 1. If $\left\|h_{1}\right\| \geq 2 A d\left\|h_{2}\right\| / \varepsilon_{0}$, then using (2.1), we obtain

$$
\begin{aligned}
\sum_{i=1}^{d}\left(\|\left(M_{z_{i}}^{*}-\bar{\lambda}_{0 i}\right)\right. & \left.h_{1}+\left(M_{z_{i}}^{*}-\bar{\lambda}_{0 i}\right) \eta h_{2} \|\right)+\left\|h_{2}\right\| \\
& \geq \sum_{i=1}^{d}\left(\left\|\left(M_{z_{i}}^{*}-\bar{\lambda}_{0 i}\right) h_{1}\right\|-\left\|\left(M_{z_{i}}^{*}-\bar{\lambda}_{0 i}\right) \eta h_{2}\right\|\right)+\left\|h_{2}\right\| \\
& \geq \varepsilon_{0}\left\|h_{1}\right\|-\frac{\varepsilon_{0}}{2}\left\|h_{1}\right\|+\left\|h_{2}\right\|=\frac{\varepsilon_{0}}{2}\left\|h_{1}\right\|+\left\|h_{2}\right\| .
\end{aligned}
$$

Noting that $\|g\|^{2}=\left\|h_{1}\right\|^{2}+\left\|h_{2}\right\|^{2}$, it is easy to see there exists $\delta_{1}>0$ independent of $g$ such that

$$
\sum_{i=1}^{d}\left\|\left(M_{z_{i}}^{*}-\bar{\lambda}_{0 i}\right) h_{1}+\left(M_{z_{i}}^{*}-\bar{\lambda}_{0 i}\right) \eta h_{2}\right\|+\left\|h_{2}\right\| \geq \delta_{1}\|g\| .
$$

CASE 2. If $\left\|h_{1}\right\| \leq 2 A d\left\|h_{2}\right\| / \varepsilon_{0}$, then

$$
\begin{aligned}
\sum_{i=1}^{d}\left(\left\|\left(M_{z_{i}}^{*}-\bar{\lambda}_{0 i}\right) h_{1}+\left(M_{z_{i}}^{*}-\bar{\lambda}_{0 i}\right) \eta h_{2}\right\|\right)+ & \left\|h_{2}\right\| \geq\left\|h_{2}\right\| \\
& \geq\left\|h_{2}\right\| / 2+\varepsilon_{0}\left\|h_{1}\right\| / 4 A d,
\end{aligned}
$$


and the same argument shows that there exists $\delta_{2}>0$ independent of $g$ such that

$$
\sum_{i=1}^{d}\left\|\left(M_{z_{i}}^{*}-\bar{\lambda}_{0 i}\right) h_{1}+\left(M_{z_{i}}^{*}-\bar{\lambda}_{0 i}\right) \eta h_{2}\right\|+\left\|h_{2}\right\| \geq \delta_{2}\|g\| .
$$

Letting $\delta=\min \left\{\delta_{1}, \delta_{2}\right\}$ proves that in both cases, the assumption (2.1) implies (2.2). Now, we show that (2.2) is not true, which implies that (2.1) is not either, and thus, $\bar{\lambda}_{0} \in \sigma\left(N_{z}^{*}\right)$ and $\lambda_{0} \in \sigma\left(N_{z}\right)$. Let us return to the beginning of the proof: there exists a sequence $\left\{\lambda_{j}\right\} \subseteq \mathbb{B}_{d}$ with $\lambda_{j}=$ $\left(\lambda_{j 1}, \ldots, \lambda_{j d}\right)$ such that

$$
\lambda_{j} \rightarrow \lambda_{0}, \quad \eta\left(\lambda_{j}\right) \rightarrow 0 \quad \text { as } j \rightarrow \infty .
$$

Let $K_{\lambda}$ be the reproducing kernel for $H^{2}\left(\mathbb{B}_{d}\right)$ and $k_{\lambda}=K_{\lambda} /\left\|K_{\lambda}\right\|$ the normalized reproducing kernel. Then

$$
\begin{aligned}
\sum_{i=1}^{d}\left\|\left(M_{z_{i}}^{*}-\bar{\lambda}_{0 i}\right) k_{\lambda_{j}}\right\|+\left\|T_{\bar{\eta}} k_{\lambda_{j}}\right\| & =\sum_{i=1}^{d}\left\|\left(\bar{\lambda}_{j i}-\bar{\lambda}_{0 i}\right) k_{\lambda_{j}}\right\|+\left\|\bar{\eta}\left(\lambda_{j}\right) k_{\lambda_{j}}\right\| \\
& =\sum_{i=1}^{d}\left|\left(\bar{\lambda}_{j i}-\bar{\lambda}_{0 i}\right)\right|+\left|\bar{\eta}\left(\lambda_{j}\right)\right| \rightarrow 0 .
\end{aligned}
$$

This contradicts (2.2), and the proof of Proposition 2.3 is complete.

It is not difficult to see that $\sigma\left(N_{z}\right) \subseteq \overline{\mathbb{B}}_{d}$. Combining Propositions 2.2 and 2.3 gives the following.

TheOREM 2.5. $\sigma\left(N_{z}\right)=Z(\eta) \cup \partial \mathbb{B}_{d}$.

3. The essential spectrum of $N_{z}$. First, we prove the following proposition.

Proposition 3.1. The quotient module $N$ is essentially normal, that $i s$, the commutators $\left[N_{z_{i}}^{*}, N_{z_{j}}\right]$ are compact for all $1 \leq i, j \leq d$.

Proof. For any $f \in L^{\infty}\left(\partial \mathbb{B}_{d}\right)$, it is easy to verify that $T_{f} T_{z_{i}}-T_{z_{i}} T_{f}$ is compact for $i=1, \ldots, d$. Since $\eta$ is an inner function, this means

$$
P_{N}=I-T_{\eta} T_{\eta}^{*}=I-T_{\eta} T_{\bar{\eta}}
$$

Since

$$
\begin{aligned}
N_{z_{i}}^{*} N_{z_{j}}-N_{z_{j}} N_{z_{i}}^{*} & =P_{N} T_{\bar{z}_{i}} P_{N} T_{z_{j}} P_{N}-P_{N} T_{z_{j}} P_{N} T_{\bar{z}_{i}} P_{N} \\
& =P_{N}\left(T_{\bar{z}_{i}} P_{N} T_{z_{j}}-T_{z_{j}} P_{N} T_{\bar{z}_{i}}\right) P_{N},
\end{aligned}
$$

inserting (3.1) and using the fact that $T_{z_{i}}$ essentially commutes with each Toeplitz operator for $i=1, \ldots, d$, we conclude that $\left[N_{z_{i}}^{*}, N_{z_{j}}\right]$ is compact for $1 \leq i, j \leq d$.

We now determine the essential spectrum $\sigma_{\mathrm{e}}\left(N_{z}\right)$. 
Lemma 3.2. $\sigma_{\mathrm{e}}\left(N_{z}\right) \subseteq \partial \mathbb{B}_{d}$.

Proof. Set $M=\eta H^{2}\left(\mathbb{B}_{d}\right)$. Since $z_{i} M \subseteq M$, we have $M_{z_{i}}^{*} N \subseteq N$ for $i=1, \ldots, d$. This means

$$
\sum_{i=1}^{d} N_{z_{i}} N_{z_{i}}^{*}=\sum_{i=1}^{d} P_{N} M_{z_{i}} P_{N} M_{z_{i}}^{*}\left|N=P_{N} \sum_{i=1}^{d} M_{z_{i}} M_{z_{i}}^{*}\right| N .
$$

Since $\sum_{i=1}^{d} M_{z_{i}} M_{z_{i}}^{*}-I$ is compact, this implies

$$
\sum_{i=1}^{d} N_{z_{i}} N_{z_{i}}^{*}=I_{N}+K
$$

where $K$ is a compact operator. By Proposition 3.1, we know the tuple $N_{z}=\left\{N_{z_{1}}, \ldots, N_{z_{d}}\right\}$ is essentially normal. Combining [Cur1, Cor. 3.10] and (3.2) gives $\sigma_{\mathrm{e}}\left(N_{z}\right) \subseteq \partial \mathbb{B}_{d}$.

LEMma 3.3. $\partial \mathbb{B}_{d} \subseteq \sigma_{\mathrm{e}}\left(N_{z}\right)$.

Proof. As in the proof of Proposition 2.3, we get a dense subset $H$ of $\partial \mathbb{B}_{d}$ such that for each $\lambda_{0} \in H$, there is a sequence $\left\{\lambda_{j}\right\} \subseteq \mathbb{B}_{d}$ such that $\lambda_{j} \rightarrow \lambda_{0}$ and $\eta\left(\lambda_{j}\right) \rightarrow 0$ as $j \rightarrow \infty$. Since $\sigma_{\mathrm{e}}\left(N_{z}\right)$ is a closed subset of $\partial \mathbb{B}_{d}$, it suffices to prove that $H \subseteq \sigma_{\mathrm{e}}\left(N_{z}\right)$.

Suppose otherwise, that is, there is a point $\lambda_{0}=\left(\lambda_{01}, \ldots, \lambda_{0 d}\right) \in H$ such that

$$
N_{z}-\lambda_{0}=\left(N_{z_{1}}-\lambda_{01}, \ldots, N_{z_{d}}-\lambda_{0 d}\right)
$$

is Fredholm. Since $\left[N_{z_{i}}, N_{z_{i}}^{*}\right]$ is compact for $1 \leq i, j \leq d$, and

$$
\left[N_{z_{i}}-\lambda_{0 i}, N_{z_{i}}^{*}-\bar{\lambda}_{0 i}\right]=\left[N_{z_{i}}, N_{z_{i}}^{*}\right]
$$

this ensures that the tuple $\left(N_{z_{1}}-\lambda_{01}, \ldots, N_{z_{d}}-\lambda_{0 d}\right)$ is essentially normal. By [Cur2, Corollary 3.9], $\left(N_{z_{1}}-\lambda_{01}, \ldots, N_{z_{d}}-\lambda_{0 d}\right)$ is Fredholm if and only if

$$
\sum_{i=1}^{d}\left(N_{z_{i}}-\lambda_{0 i}\right)\left(N_{z_{i}}^{*}-\bar{\lambda}_{0 i}\right)
$$

is Fredholm. Since this last operator is positive, there exist an invertible positive operator $A$ and a compact operator $K$ such that

$$
\sum_{i=1}^{d}\left(N_{z_{i}}-\lambda_{0 i}\right)\left(N_{z_{i}}^{*}-\bar{\lambda}_{0 i}\right)=A+K .
$$

Recalling that $k_{\lambda}$ is the normalized reproducing kernel of $H^{2}\left(\mathbb{B}_{d}\right)$, we have

$$
P_{N} k_{\lambda}=\left(I-M_{\eta} M_{\eta}^{*}\right) k_{\lambda}=k_{\lambda}-\overline{\eta(\lambda)} \eta k_{\lambda} .
$$


Assume $\lambda=\left(\lambda_{1}, \ldots, \lambda_{d}\right)$. Then

$$
\begin{aligned}
\sum_{i=1}^{d}\left(\left\langle\left(N_{z_{i}}-\right.\right.\right. & \left.\left.\left.\lambda_{0 i}\right)\left(N_{z_{i}}^{*}-\bar{\lambda}_{0 i}\right) P_{N} k_{\lambda}, P_{N} k_{\lambda}\right\rangle\right)^{1 / 2} \\
& =\sum_{i=1}^{d}\left\|M_{z_{i}-\lambda_{0 i}}^{*} P_{N} k_{\lambda}\right\|=\sum_{i=1}^{d}\left\|M_{z_{i}-\lambda_{0 i}}^{*}\left(k_{\lambda}-\overline{\eta(\lambda)} \eta k_{\lambda}\right)\right\| \\
& =\sum_{i=1}^{d}\left\|\overline{\lambda_{i}-\lambda_{0 i}} k_{\lambda}-\overline{\eta(\lambda)} M_{z_{i}-\lambda_{0 i}}^{*} \eta k_{\lambda}\right\| \\
& \leq \sum_{i=1}^{d}\left(\left|\lambda_{i}-\lambda_{0 i}\right|+|\overline{\eta(\lambda)}|\left\|M_{z_{i}-\lambda_{0 i}}^{*} \eta k_{\lambda}\right\|\right) .
\end{aligned}
$$

Replacing $\lambda$ in (3.5) with $\lambda_{j}=\left(\lambda_{j 1}, \ldots, \lambda_{j d}\right)$ and noting $\left\|\eta k_{\lambda}\right\|=1$ and $\eta\left(\lambda_{j}\right) \rightarrow 0$, we see that the last two terms in (3.5) converge to 0 as $j \rightarrow \infty$. Thus we have

$$
\sum_{i=1}^{d}\left\langle\left(N_{z_{i}}-\lambda_{0 i}\right)\left(N_{z_{i}}^{*}-\bar{\lambda}_{0 i}\right) P_{N} k_{\lambda_{j}}, P_{N} k_{\lambda_{j}}\right\rangle \rightarrow 0 \quad \text { as } j \rightarrow \infty .
$$

On the other hand,

$$
\begin{aligned}
& \left\langle(A+K) P_{N} k_{\lambda}, P_{N} k_{\lambda}\right\rangle \\
& \quad=\left\langle A\left(k_{\lambda}-\overline{\eta(\lambda)} \eta k_{\lambda}\right), k_{\lambda}-\overline{\eta(\lambda)} \eta k_{\lambda}\right\rangle+\left\langle K\left(k_{\lambda}-\overline{\eta(\lambda)} \eta k_{\lambda}\right), k_{\lambda}-\overline{\eta(\lambda)} \eta k_{\lambda}\right\rangle .
\end{aligned}
$$

We compute the two terms on the right of the above equality:

$$
\begin{aligned}
& \left\langle A\left(k_{\lambda}-\overline{\eta(\lambda)} \eta k_{\lambda}\right), k_{\lambda}-\overline{\eta(\lambda)} \eta k_{\lambda}\right\rangle \\
& =\left\langle A k_{\lambda}, k_{\lambda}\right\rangle-\overline{\eta(\lambda)}\left\langle A \eta k_{\lambda}, k_{\lambda}\right\rangle-\eta(\lambda)\left\langle A k_{\lambda}, \eta k_{\lambda}\right\rangle+|\eta(\lambda)|^{2}\left\langle A \eta k_{\lambda}, \eta k_{\lambda}\right\rangle .
\end{aligned}
$$

Note that $A$ is an invertible positive operator, hence so is $\sqrt{A}$. Thus $\sqrt{A}$ is bounded below, i.e. there is $c>0$ such that $\|\sqrt{A} f\| \geq c\|f\|$ for any $f \in N$. Therefore,

$$
\left\langle A k_{\lambda}, k_{\lambda}\right\rangle=\left\|\sqrt{A} k_{\lambda}\right\|^{2} \geq c^{2} .
$$

With $\lambda$ replaced by $\lambda_{j}$, we conclude that the other three terms of the last equality in (3.7) converge to 0 as $j \rightarrow \infty$. So we have

$$
\left\langle A P_{N} k_{\lambda_{j}}, P_{N} k_{\lambda_{j}}\right\rangle \geq c^{2} .
$$

Moreover, since $P_{N} k_{\lambda_{j}} \stackrel{w}{\rightarrow} 0$, and $K$ is compact, we have

$$
\left\langle K P_{N} k_{\lambda_{j}}, P_{N} k_{\lambda_{j}}\right\rangle \rightarrow 0 \quad \text { as } j \rightarrow \infty .
$$

Combining (3.3), (3.6), (3.8), and (3.9), we get a contradiction. Therefore, $\partial \mathbb{B}_{d} \subseteq \sigma_{\mathrm{e}}\left(N_{z}\right)$.

From Lemmas 3.2 and 3.3, we have 
Theorem 3.4. $\sigma_{\mathrm{e}}\left(N_{z}\right)=\partial \mathbb{B}_{d}$.

The next proposition comes from a discussion with R. Yang.

Proposition 3.5. The $C^{*}$-algebra $C^{*}\left(N_{z}\right)$ generated by $\left\{N_{z_{1}}, \ldots, N_{z_{d}}, I\right\}$ is irreducible, that is, if $Q$ is a projection on $N$ and $Q N_{z_{i}}=N_{z_{i}} Q$ for $i=1, \ldots, d$, then $Q=0$ or $Q=I$.

Proof. From the proof of Proposition 2.2 in [GZ], there exist polynomials $p_{i}, q_{i}$ such that

$$
1 \otimes 1=\sum_{i=1}^{m} M_{p_{i}} M_{q_{i}}^{*} \quad \text { on } H^{2}\left(\mathbb{B}_{d}\right) .
$$

Set $e=P_{N} 1$. By (3.10), we have

$$
e \otimes e=P_{N}(1 \otimes 1) P_{N}=\sum_{i=1}^{m} P_{N} M_{p_{i}} M_{q_{i}}^{*} P_{N}=\sum_{i=1}^{m} N_{p_{i}} N_{q_{i}}^{*},
$$

where, for a polynomial $p, N_{p}=P_{N} M_{p} \mid N=p\left(N_{z_{i}}\right)$. If $Q$ is a projection as in the hypothesis, then $Q N_{q}=N_{q} Q$ and $Q N_{q}^{*}=N_{q}^{*} Q$ for any polynomial $q$. Thus, $Q(e \otimes e)=(e \otimes e) Q$, which implies

$$
Q e \otimes e=e \otimes Q e .
$$

Making both sides of the above equality act on $e$, we have

$$
Q e=\frac{\|Q e\|^{2}}{\|e\|^{2}} e .
$$

CASE 1. If $Q e \neq 0$, then

$$
Q e=Q^{2} e=\frac{\|Q e\|^{4}}{\|e\|^{4}} e=\frac{\|Q e\|^{2}}{\|e\|^{2}} e .
$$

This implies $\|Q e\|=\|e\|$, hence $Q e=e$. Now for any $f \in N$, there exists a sequence of polynomials $q_{n}$ such that $q_{n} \rightarrow f$ as $n \rightarrow \infty$. Writing $1=e+\xi$ with $\xi \in \eta H^{2}\left(\mathbb{B}_{d}\right)$, we have

$$
q_{n}=q_{n}(e+\xi) \rightarrow f \quad \text { as } n \rightarrow \infty .
$$

Hence,

$$
P_{N}\left(q_{n} e+q_{n} \xi\right)=P_{N} q_{n} e \rightarrow f,
$$

that is, $N_{q_{n}} e \rightarrow f$. Therefore, $Q N_{q_{n}} e \rightarrow Q f$. Moreover, since

$$
Q N_{q_{n}} e=N_{q_{n}} Q e=N_{q_{n}} e \rightarrow f,
$$

we obtain $Q f=f$, which shows $Q=I$.

CASE 2. If $Q e=0$, the same reasoning gives $Q=0$, and the proof is complete.

Since $C^{*}\left(N_{z}\right)$ is irreducible, and $C^{*}\left(N_{z}\right) \cap \mathcal{K}(N) \neq \emptyset$ from (3.2), where $\mathcal{K}(N)$ denotes the compact operator ideal on $N$, this implies $C^{*}\left(N_{z}\right) \supseteq \mathcal{K}(N)$ 
by [Arv4]. Since the tuple $N_{z}$ is essentially normal, we have the following exact sequence:

$$
0 \rightarrow \mathcal{K}(N) \hookrightarrow C^{*}\left(N_{z}\right) \stackrel{\pi}{\rightarrow} C\left(\partial \mathbb{B}_{d}\right) \rightarrow 0,
$$

where $\pi$ is the unital $*$-homomorphism given by $\pi\left(N_{z_{i}}\right)=Z_{i}$.

From $[\mathrm{BDF}]$, the above exact sequence gives an extension of $\mathcal{K}(N)$ by $C\left(\partial \mathbb{B}_{d}\right)$.

Proposition 3.6. The short exact sequence (3.12) is split, that is, there exists a *-homomorphism $\sigma: C\left(\partial \mathbb{B}_{d}\right) \rightarrow C^{*}\left(N_{z}\right)$ such that $\pi \sigma=I$.

Proof. It follows from Theorem 3.4 that the tuple $\left(N_{z_{1}}, \ldots, N_{z_{d}}\right)$ is Fredholm. Moreover, by [GRS, Corollary 3.5] the tuple has Fredholm index 0 . Since $\operatorname{Ext}\left(\partial \mathbb{B}_{d}\right)=K_{1}\left(\partial \mathbb{B}_{d}\right)=\mathbb{Z}$, the short exact sequence (3.12) defines a zero element in $K_{1}\left(\partial \mathbb{B}_{d}\right)$. Indeed, this $K$-homology element is determined by the index of the tuple $\left(N_{z_{1}}, \ldots, N_{z_{d}}\right)$ (cf. [BDF, Guo2]) and thus is 0 . So the extension defined by (3.12) is a trivial element in $\operatorname{Ext}\left(\partial \mathbb{B}_{d}\right)$. This means that the short exact sequence (3.12) is split.

REMARK. In the cases $d=2,3$, the extensions defined by quotients of submodules generated by homogeneous polynomials are not split [GW].

\section{References}

[Arv1] W. Arveson, Subalgebras of $C^{*}$-algebras, Acta Math. 123 (1969), 141-224.

[Arv2] -, Subalgebras of $C^{*}$-algebras III: Multivariable operator theory, ibid. 181 (1998), 159-228.

[Arv3] -, p-Summable commutators in dimension d, J. Operator Theory 54 (2005), 101-117.

[Arv4] -, An Invitation to $C^{*}$-Algebras, Springer, 1976.

[Arv5] -, The Dirac operator of a commuting d-tuple, J. Funct. Anal. 189 (2002), 53-79.

[Arv6] -, Quotients of standard Hilbert modules, arXiv: math. OA/0502388, v2, 14. Jul, 2005.

[BDF] L. Brown, R. Douglas and P. Fillmore, Extension of $C^{*}$-algebras and K-homology, Ann. of Math. 105 (1977), 265-324.

[CG] X. M. Chen and K. Y. Guo, Analytic Hilbert Modules, Chapman \& Hall/CRC Res. Notes in Math. 433, Chapman \& Hall/CRL, 2003.

[Cur1] R. Curto, Fredholm and invertible n-tuples of operators. The deformation problem, Trans. Amer. Math. Soc. 266 (1981), 129-159.

[Cur2] -, Applications of several complex variables to multiparameter spectral theory, in: Surveys of Some Results in Operator Theory, J. B. Conway and B. B. Morrel (eds.), Pitman Res. Notes Math. Ser. 192, 1988, 25-90.

[Dou] R. Douglas, Essentially reductive Hilbert modules, J. Operator Theory 55 (2006), $117-133$.

[DP] R. Douglas and V. Paulsen, Hilbert Modules over Function Algebras, Pitman Res. Notes Math. Ser. 217, Longman, 1989. 
[GRS] J. Gleason, S. Richter, and C. Sundberg, On the index of invariant subspaces in spaces of analytic functions of several complex variables, J. Reine Angew. Math. 587 (2005), 49-76.

[Guo1] K. Y. Guo, Defect operators for submodules of $H_{d}^{2}$, ibid. 573 (2004), 181-209.

[Guo2] -, Essentially commutative $C^{*}$-algebras with essential spectrum homeomorphic to $S^{2 n-1}$, J. Austral. Math. Soc. 70 (2001), 199-210.

[GW] K. Y. Guo and K. Wang, Essentially normal Hilbert modules and K-homology, preprint.

[GZ] K. Y. Guo and D. C. Zheng, Invariant subspaces, quasi-invariant subspaces, and Hankel operators, J. Funct. Anal. 187 (2001), 308-342.

[Nil] N. Nikol'skiı̌, Treatise on the Shift Operator (Spectral Function Theory), Grundlehren Math. Wiss. 273, Springer, 1986.

[Rud1] W. Rudin, Function Theory in the Unit Ball of $\mathbb{C}^{n}$, Grundlehren Math. Wiss. 241, Springer, 1980.

[Rud2] - New constructions of functions holomorphic in the unit ball of $\mathbb{C}^{n}$, CBMS Reg. Conf. Ser. Math. 63, Amer. Math. Soc., 1986.

[Tay] J. Taylor, A joint spectrum for several commuting operators, J. Funct. Anal. 6 (1970), 172-191.

Department of Mathematics

Fudan University

Shanghai, 200433, P.R. China

E-mail: kyguo@fudan.edu.cn

031018011@fudan.edu.cn

Received September 30, 2005

Revised version September 11, 2006 